\title{
Internal Configuration of Prismatic Lithium-ion Cells at the Onset of Mechanically Induced Short Circuit
}

\author{
Hsin Wang ${ }^{\text {a }}$, Srdjan Simunovic ${ }^{\text {a }}$, Hossien Maleki ${ }^{\mathrm{b}}$, Jason N. Howard ${ }^{\mathrm{b}}$ and Jeraled A. Hallmark ${ }^{\mathrm{b}}$ \\ ${ }^{a}$ Oak Ridge National Laboratory, Oak Ridge, TN 37831, USA \\ ${ }^{b}$ Motorola Mobility, 1700 Belle Meade Court, Lawrenceville, GA 30043, USA
}

\begin{abstract}
The response of Li-ion cells to mechanically induced internal electrical shorts is an important safety performance metric design. We assume that the battery internal configuration at the onset of electrical short influences the subsequent response and can be used to gage the safety risk. We subjected a series of prismatic Li-ion cells to lateral pinching using $0.25 ", 0.5 ", 1$ ", 2 " and 3" diameter steel balls until the onset of internal short. The external aluminum enclosure froze the internal cell configuration at the onset of short and enabled us to cross-section the cells, and take the cross-section images.. The images indicate that an internal electric short is preceded by extensive strain partitioning in the cells, fracturing and tearing of the current collectors, cracking and slipping of the electrode layers with multiple fault lines across multiple layers. These observations are at odds with common notion of homogeneous deformation across the layers and strain hardening of electrodes that eventually punch through the separator and short the cell. The faults are akin to tectonic movements of multiple layers that are characteristic of granular materials and bonded aggregates. The short circuits occur after extensive internal faulting, which implies significant stretching and tearing of separators.
\end{abstract}

Keywords: Li-ion cells, mechanical deformation

Corresponding author e-mail address: wangh2@ornl.gov, Tel: 865-576-5074, FAX: 865-574-6098 


\section{Introduction}

Li-ion batteries have been widely used to power portable electronic devices. Recently, Li-ion batteries have transitioned from prototype to commercial electric vehicles [1], where vehicle crashworthiness and safety are major design considerations. Vehicle crash can expose batteries to intense deformation and accelerations so that battery response under dynamic loading must be included in the overall safety assessment [2]. Unlike portable electronics devices which use a small number of low capacity cells, electrical vehicles can use hundreds of large-format, tightlypacked, high-capacity cells. As the numbers of electric vehicles increase on the road, the chances of accidents proportionally increase. Li-ion batteries and the supporting structures must be designed to remain safe when subjected to high-speed impacts. Lacking an in-depth understanding of how the batteries fail under mechanical deformation, the current approach is to protect the batteries by heavy, armor-like enclosures [3]. This adds significant weight to the vehicle and reduces its range and energy efficiency. Even with a heavy protection, batteries can still deform under extreme crash conditions and understanding of the safety risks associated with deformation is needed.

Various tests have been developed to evaluate the safety of Li-ion cells under mechanical deformation. The techniques to induce internal short circuit on fully assembled cells are nail penetration [4], indentation [5, 6] and pinch $[7,8]$ tests. The purpose of these tests is to create a small break in the separator mimicking the very rare internal short circuit events in Li-ion cells. Mechanical abuse tests of the Li-ion cells include dropping [9, 10], and crushing[11]. These tests focus on the overall cell response to mechanical deformations and the subsequent thermal events. No experiments have been reported in the literature for investigating the detailed mechanisms of 
internal cell deformation and configuration leading to internal shorts due to lateral mechanical deformation. Crush and impact tests have also been carried out for cells, modules and packs used in electric vehicles [11]. These tests focus on battery response and safety risks after mechanical deformation. The test results, safety outcomes and ranking of thermal events are usually based on the USABC tables [12].

The main reasons for the lack of mapping of the kinematic processes of internal mechanical deformation of the battery cells are the destructive nature of the mechanical abuse tests and the difficulties associated with freezing the battery state. Since the final outcome is usually a short circuit, thermal runaway or fire, there is often very little evidence left to examine. Even for tests that do not end with thermal runaway, local heating can melt the separator(s) making post-mortem interpretation of the samples very difficult. Jellyrolls are kept under compression in cells and have significant spring-back when the cell casing is removed. Opening the cells after mechanical deformation and peeling each layer to find and expose the origin of failure eliminates much of the in-situ deformation information. For most pouch cells, the original deformation cannot be retained as soon as the applied force is removed because of the combination of the spring back of the jellyroll and the flexibility of the pouch enclosure.

Li-ion cells have been studied non-destructively using X-ray tomography $[13,14]$ and neutron imaging $[15,16]$. The cost, setup constraints and interpretation of the data limit their effectiveness and practicality. 
In this paper, we report on a procedure to study the internal configuration of Li-ion cells at the onset of electrical short due to mechanical loading. Commercially available $\mathrm{LiCoO}_{2}$ prismatic Liion cells with $800 \mathrm{mAhr}$ state of charge in aluminum cans were subjected to lateral mechanical pinch. The cell capacity was kept at minimum to avoid thermal damage and a voltage drop was used as the indicator of the onset of internal short circuit. The aluminum enclosures of the cells froze the internal mechanical deformation after the removal of external load. The deformed cells were discharged and cut using a slow-speed diamond saw at the center of indentation. The crosssectional areas were then imaged using optical microscopy. This technique allowed us to investigate the actual kinematic processes of deformation inside the Li-ion cells. This study gives the first-hand information about the prevailing internal kinematic deformation mechanisms of Liion cell and suggests directions for modeling of the jellyroll response and the onset of short circuit

\section{Experimental setup}

The pinch test configuration was similar to previously reported tests [7] for internal short circuit simulation. The Li-ion cells used are commercially available $\mathrm{LiCoO}_{2}$ batteries with dimensions of $34 \times 42 \times 4.3 \mathrm{~mm}$ and capacity of $800 \mathrm{mAhr}$. Fig. 1(a) shows a schematic of the pinch test setup. The two steel spheres shown in Fig. 1(a) were used to apply concentrated coaxial loading on both sides (top and bottom) of a prismatic cell. The spheres were connected with rigid rods to the actuator and load cell of a servo hydraulic mechanical testing machine (MTS Systems with model 407 controller). Stainless steel spheres of diameters between 0.25 inch and 3 inch were used.

Internal short circuits were typically detected at $>60 \%$ reduction of the total thickness. A polycarbonate box was used to contain fragments in case a cell went into thermal runaway during the test and was connected to the laboratory's ventilation system. The tests were carried out under 
constant speed controlled by the machine's actuator. The cell open circuit voltage $\left(V_{\text {ocv }}\right)$ was used as feedback to a computer controlling operation of the servo hydraulic testing machine. Whenever the cell open circuit voltage $V_{\text {ocv }}$ dropped below a preset threshold value, i.e. $0.10 \mathrm{~V}$, the motion was stopped and the moving sphere (the other sphere was stationary) returned to its initial position. Various control parameters, such as sphere size, loading speed, stroke response mode (hold or return when the $V_{\text {ocv }}$ of a cell drops below a preset threshold voltage), and the preset threshold voltage, can be adjusted. The picture in Fig. 1(b) shows a Li-ion cell after pinching with 0.5 " diameter spheres. Figure 2 shows the results of a typical pinch test using 1-inch diameter steel balls. The battery was charged to $4.0 \mathrm{~V}$ and short circuit occurred at 64.2 second with maximum load of 1947 pounds and displacement of -0.064 inch $(-1.65 \mathrm{~mm})$.

\section{Results and discussion}

\subsection{Mechanical deformation of Li-ion cells using 0.25 ", 0.5 ", 1", 2" and 3" diameter} spheres

Mechanical deformation of the internal layers of Li-ion cell after pinch tests using 0.25 ", 0.5 ", 1.0", 2.0" and 3.0" diameter spheres are shown in Figures 3 (a) - (e). The following kinematic features of deformation were observed:

- Tearing of current collectors: Current collectors under the indenter are discontinuous, showing evidence of cracking and tearing.

- Through layer cracking (fault lines): In Figures 3(a), (b) and (c), shear-type faults span multiple-layers and are oriented at approximately a $45^{\circ}$ angle. 
- Kinking of layers: Correlated kinking extending for multiple layers from the indenter can be seen in Figures 3(a)-(e).

- Local melting: Small black voids without the original materials were observed in Figure 2(e).

Figure 4 shows close-up views of the Li-ion cell after a pinch test using 0.25 " spheres. The current collectors beneath the aluminum can in Figure 4(a)-(b) are torn apart by several $45^{\circ}$ angle faults. The cross section images show the current collector fragments between $200 \mu \mathrm{m}$ to $700 \mu \mathrm{m}$ indicating multiple, possibly radial, cracks from the center of the indentation. In Figure 4(b), the copper and aluminum foils on opposite sides of shear faults are separated by s significant amount. Electrode materials previously contained between the current collectors were able to "flow" into the faults pushing the torn current collectors to deform along with the separators. The distribution and curvature of the current collector act as tracers of deformation and movement. Folding of the layer and kinks are shown clearly in Figure 4(c).

The kinking of the electrodes near the edge of the cell-can, in Figures 4 (b) and (c), shows cell edge deformation under mechanical abuse. It is one of the main modes of internal short circuit under soft mechanical deformation (e.g. drop on the edge), in which separator tends to fold back into itself causing anode-cathode micro-shorting. This failure mode often shows a latent effect (battery self-discharge).

Figure 5 shows close up images of a cell after a pinch test using 0.5 " diameter spheres. The smaller curvature of the spheres caused more homogeneous deformation of the electrode material layers. However, shear faults still dominate the global deformation, as shown by a $45^{\circ}$ fault line 
across multiple layers in Figure 5(a). The close up view in Figure 5(b) shows the crack propagation through the layers and movement of the electrode materials along with the separator into the fault.

Figure 6 shows internal deformation imparted by pinch spheres of 1 " in diameter at the onset of internal short circuit. The fault lines span across multiple layers of current collectors that were torn apart along with the electrode materials, Figure 6(a). Figure 6(b) shows substantial relocation of electrode materials. Materials were pushed downward and several current collectors slid along the fault line. The separators were stretched in conjunction with electrode materials. In the middle of the image in Fig. 6(b), torn current collectors were separated only by the separator with no electrode materials. In the upper area of the image, anode and cathode materials were only separated by the separator with no current collectors. In both cases, the separators were stretched and no short circuit was observed in the area with material movements.

Figure 7 shows cross-section images of a pinched cell using 2" diameter spheres at the onset of internal short. Wave-like movement in the layers can be clearly seen. Notable is the absence of fault lines compared to pinching with smaller diameters which does not exclude their occurrence in other cross sections. Figure 8 is an image of a pinch test using 3" diameter spheres. The tearing of the current collectors and shear faults are again prominent features of the deformation. The tearing triggered fault through the layers and shifting of the electrode materials. Spacing between the layers also started to decrease. The stresses on the current collectors were released, with increased stretching of the separators. 
From the cross section images of the layers we postulate the following steps in jellyroll response to crushing:

(a) Initial homogeneous compression of the jellyroll: Deformation is distributed according to the compliance of each layer. The displacement field is homogeneous and determined by the shape of the indenter and the overall anisotropy of the jellyroll.

(b) Fault formation: Increased loading eventually exceeds the strength of the jellyroll resulting in a localized fault formation. The exact process of the fault formation is yet to be discerned. One likely scenario indicated by the approximately 45 degree fault angle, is that the low shear strength of electrode materials triggers shear localization in several electrode layers which in turn localizes tearing of the current collectors in between.

(c) Materials flow and internal rearrangement: Electrode materials near the fault line will flow around the torn current collectors and may slide into the fault. Since control of the external loading is based on the rearrangement of material may temporarily relieve the external force. Further indentation may eventually lead to a repeat of the above two steps.

(d) Separator failure: Separators are stretched and twisted to conform to the faults and eventually exceed the failure point, resulting in internal short circuits as opposite electrode materials and/or the pieces of current collectors come into contact.

\subsection{Experiment interpretation and possible mechanisms of deformation}

For compressive loading orthogonal to the plane of the jellyroll, the commonly assumed sequence of deformation mechanisms and events leading to the internal short is conceptually simple $[7,17$, 
18]. The compression creates a continuous displacement field and distributes through-thickness deformation according to the compliance of each layer [19]. With continuous indentation, deformation localizes into three successive cell layers (electrodes, separator) closest to the indenter. The separator layer eventually fails by intrusion of deformation-hardened electrodes and exposes the electrodes of opposite polarity to each other thereby creating an electrical short. The above scenario is reinforced by models of metals plasticity which are usually the first choice when encountering nonlinear deformation.

While there is evidence of the above scenario for a single or few layers of a jellyroll [7], our experiments on cells with many layers indicate a different deformation mode and sequence of events. The experiments clearly show strain partitioning and segmentation. The electric shorts occur after extensive, multi-layer shear faults and fractures in the jellyroll instead of a single pinpoint failure brought about by global homogeneous compression. The faults are akin to tectonic movements [20] across multiple layers [21, 22] typical of bonded aggregates [22]. The short circuits occur after extensive internal faulting, which implies large stretching and tearing of separators. The shear faults imply that electrode materials may have a significant effect, which seems reasonable since they can account for $80 \%$ of the jellyroll [23]. The electrode material is a composite of active particles glued into a solid skeleton with its interstices saturated by electrolyte. With porosity of about $30 \%$ [23], this composite is much more similar to saturated cohesive granular soils and bonded aggregates than to plastic metallic materials. The similarity between the formation of multi-layer shear faults in cell electrode materials and in soils is also indicative that the concepts and tools from mechanics of soils and bonded aggregates can be expected to offer a 
framework for understanding the internal battery deformation. The current collector foils can then be viewed as reinforcing membranes or shells that increase the resistance to shear faulting.

When the external loading induces in-plane compression of the jellyroll, the deformation exhibits even more complex features. In our tests, the shear fault fragmentations have triggered internal rearrangements which push the jellyroll segments in its plane. This in-plane deformation also exhibits some features of layered soils [24, 25], but without localized shear faults across the jellyroll. The current collectors synchronously bend and fold by off-axis compression and provide continuous containment of the electrochemical assembly. Under in-plane compression, the current collectors bend but do not break until very large deformations since bending has a stabilizing effect on foil cross sections. The bending radius is bounded by the thickness of the electrodes, so that sharp kinks do not form initially. The bending of metals allows for much higher surface strains than the uniaxial loading, and such behavior is clearly demonstrated by the in-plane compressive deformation of battery jellyrolls $[9,26]$. Continuous jamming and sharpening of the cusps may eventually lead to tearing or puncture of the separator and an electrical short.

Our battery indentation experiments indicate that current collectors are acting as embedded membrane or shell reinforcements. For the loading orthogonal to the jellyroll, the experiments show formation of a shear slip band across several layers. A possible mechanism for this cooperative fault could be an occurrence of a shear slip in the electrode material and the accompanying tensile/tear failure in the current collector. As the shear slip (or crack) in the active material reaches a metal foil, it is likely that it localizes the tensile stresses in the foil. The shear band formation in bonded aggregates is usually accompanied with dilation in the shear fault which 
can accentuate tension in the foils. The strength of the bond between active material and the binder is higher than the bond between the active material and the foil [27] which can also influence the local force transfer between the components [28]. The foils are low hardening materials, so that in uniaxial tension, their deformation is expected to localize into a thin band whose width is proportional to the foil thickness. With increased stretching, the band widens and the local thickness rapidly reduces. When the band widens to about twice the thickness, thickness reduces to zero and the load carrying of the foil vanishes. The tip configuration of the foil fragments in the polished cross-sections indicates tensile failures.

Due to its porous microstructure and mechanical properties [29, 30], the separator probably does not strongly contribute to the structural response of the jellyroll. It can stretch much more than metal foils $[31,32]$ so we can expect it to break after them, creating an electrical short. For inplane compression, crumpling of the edges of the jellyroll, especially near the tabs, is more likely to perforate the separator, as indicated by experiments. Coordinated fold zones tend to separate electrode materials at the fold cusps and as long it is more energetically favorable, new folds are created in the jellyroll. Continuity of the current collectors implies continuity of the separator because of almost an order of magnitude larger ductility of the latter. The progressive, threedimensional folding eventually leads to geometrical lockup of the folded jellyroll [26], and can create tears in the separator locally by compressing it against increasingly sharpened features caused by deformation.

Clearly, there is no shortage of mechanically induced internal short scenarios and our interpretations hinge on a limited set of experiments and battery form factors. The practical 
difficulties with the cell deformation measurements and experimental scatter can be hopefully be alleviated by postulating different mechanisms of deformation and comparing the simulations with experiments.

\section{Conclusions}

Pinching tests of prismatic Li-ion cells until onset of internal short circuit show large localized deformations and rearrangements in the jellyroll. The permanent deformation of cells' aluminum casings freeze the internal configuration of the jellyroll at the onset of short and support the samples during cross-sectioning and imaging. The test method enables investigation of underlying kinematic processes of deformation and mapping of configuration of internal electrical shorts. In our experiments, the deformation in jellyroll show shear fault zones that span multiple layers, tearing of current collectors, rearrangement of fragments, and flow of electrode materials into the faults. The similarities with deformation characteristics of granular layered solids and bonded aggregates suggest that active materials in the jellyroll have much more significant role than previously assumed.

\section{Acknowledgements}

The authors would like to thank Wei Cai, Tom Geer, Jun Qu and Donald L. Erdman III for their supports on testing and sample preparations. They would also acknowledge the support of Laboratory Director's Research and Development (LDRD) program. Oak Ridge National Laboratory (ORNL) is managed by the UT-Battelle LLC, for the Department of Energy under contract DE-AC05000OR22725. 


\section{References}

[1] M.S. Alam, Advances in Automobile Engineering, 2 (2013).

[2] D. Doughty, E.P. Roth, The Electrochemical Society Interface, 21 (2012) 37-44.

[3] L. Hollmotz, M. Hackmann, in: 22nd International Technical Conference on Enhanced Safety of Vehicles (ESV), 2011, pp. 11-0269.

[4] J.P. Peres, F. Perton, C. Audry, P. Biensan, A. de Guibert, G. Blanc, M. Broussely, Journal of Power Sources, 97-8 (2001) 702-710.

[5] L. Florence, in: Battery Power, Dallas, Texas, 2010, pp. 576.

[6] UL, in: UL 1642 Standard for Safety for Lithium Batteries, UL, 1999.

[7] W. Cai, H. Wang, H. Maleki, J. Howard, E. Lara-Curzio, Journal of Power Sources, 196 (2011) 7779-7783.

[8] F. Ren, T. Cox, H. Wang, Journal of Power Sources, 249 (2014) 156-162.

[9] E. Sahraei, R. Hill, T. Wierzbicki, Journal of Power Sources, 201 (2012) 307-321.

[10] R. Spotnitz, R. Muller, The Electrochemical Society Interface, 12 (2012) 57-60.

[11] T. Wierzbicki, E. Sahraei, Journal of Power Sources, 241 (2013) 467-476.

[12] C.N. Ashtiani, ECS Transactions, 11 (2008) 1-11.

[13] P. Shearing, R. Bradley, J. Gelb, N. Brandon, P. P Withers, Microscopy and Microanalysis, $17(2011) 1672-1673$

[14] M. Ebner, F. Geldmacher, F. Marone, M. Stampanoni, V. Wood, Advanced Energy Materials, 3 (2013) 845-850.

[15] J.B. Siegel, X.F. Lin, A.G. Stefanopoulou, D.S. Hussey, D.L. Jacobson, D. Gorsich, Journal of the Electrochemical Society, 158 (2011) A523-A529. 
[16] J. Banhart, A. Borbely, K. Dzieciol, F. Garcia-Moreno, I. Manke, N. Kardjilov, A.R. KaysserPyzalla, M. Strobl, W. Treimer, International Journal of Materials Research, 101 (2010) 10691079.

[17] H. Maleki, J.N. Howard, Journal of Power Sources, 191 (2009) 568-574.

[18] P. Ramadass, W.F. Fang, Z.M. Zhang, Journal of Power Sources, 248 (2014) 769-776.

[19] Y.-L. Shen, Constrained Deformation of Materials Devices, Heterogeneous Structures and Thermo-Mechanical Modeling, Springer, 2010.

[20] C.W. Passchier, R.A.J. Trouw, Microtectonics, Springer, 2005.

[21] D.D. Pollard, R.C. Fletcher, Fundamentals of Structural Geology, Cambridge University Press, 2010.

[22] T.W. Kidan, J.W. Cosgrove, Journal of Structural Geology, 18 (1996) 461-474.

[23] C. Glaize, S. Genies, Lithium Batteries and other Electrochemical Storage Systems, WileyISTE, 2013.

[24] R. Edmunds, G.W. Hunt, M.A. Wadee, Journal of the Mechanics and Physics of Solids, 54 (2006) 384-400.

[25] R. Edmunds, B.J. Hicks, G. Mullineux, Journal of Structural Geology, 32 (2010) 997-1008.

[26] M.Y. Ali, W.J. Lai, J. Pan, Journal of Power Sources, 242 (2013) 325-340.

[27] J.C. Chen, J.Y. Liu, Y. Qi, T. Sun, X.D. Li, Journal of the Electrochemical Society, 160 (2013) A1502-A1509.

[28] Z. Suo, C.F. Shih, Models for Metal/Ceramic Interface Fracture, in: S. Suresh, A. Mortensen, A. Needleman (Eds.) Fundamentals of Metal-Matrix Composites, Butterworth-Heinemann, 1993. [29] P. Arora, Z.M. Zhang, Chemical Reviews, 104 (2004) 4419-4462.

[30] S.S. Zhang, Journal of Power Sources, 164 (2007) 351-364. 
[31] J. Cannarella, X.Y. Liu, C.Z. Leng, P.D. Sinko, G.Y. Gor, C.B. Arnold, Journal of the Electrochemical Society, 161 (2014) F3117-F3122.

[32] J.C. Chen, Y.D. Yan, T. Sun, Y. Qi, X.D. Li, Rsc Advances, 4 (2014) 14904-14914. 


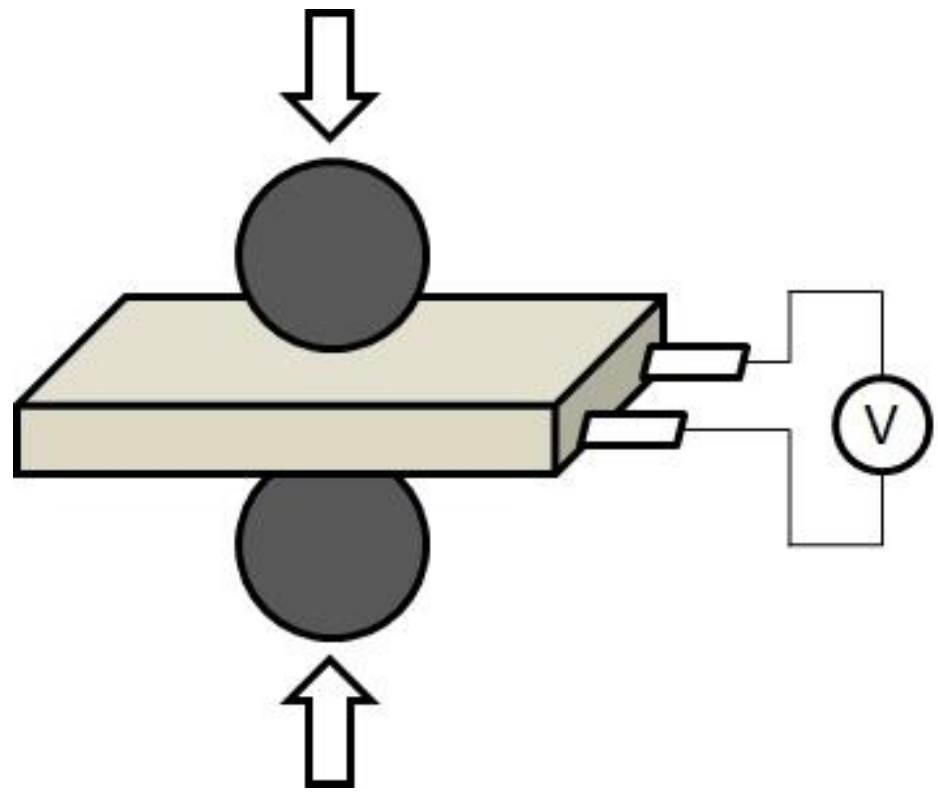

(a)

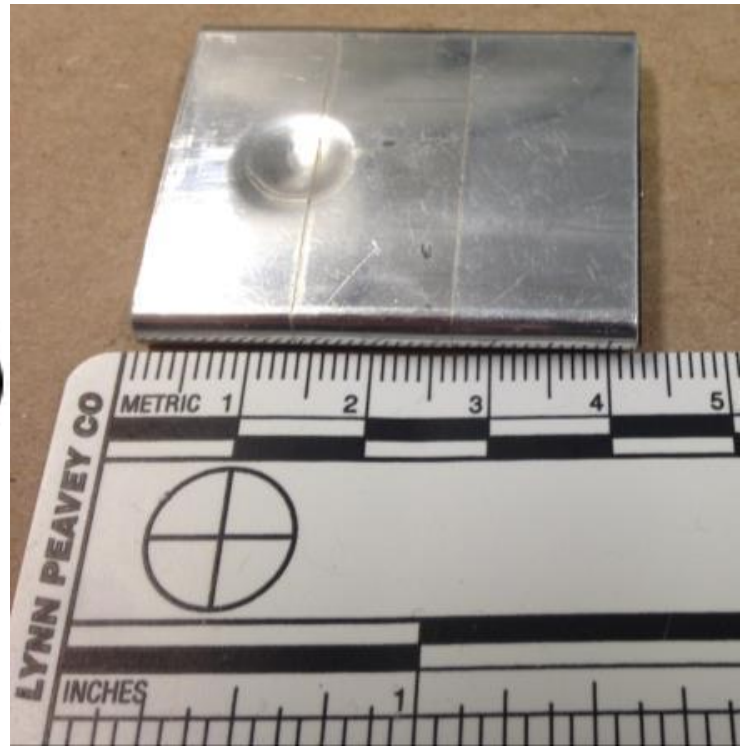

(b)

Fig. 1. (a) Schematic of Li-ion cell pinch test setup, (b) Picture of a pinched prismatic cell 


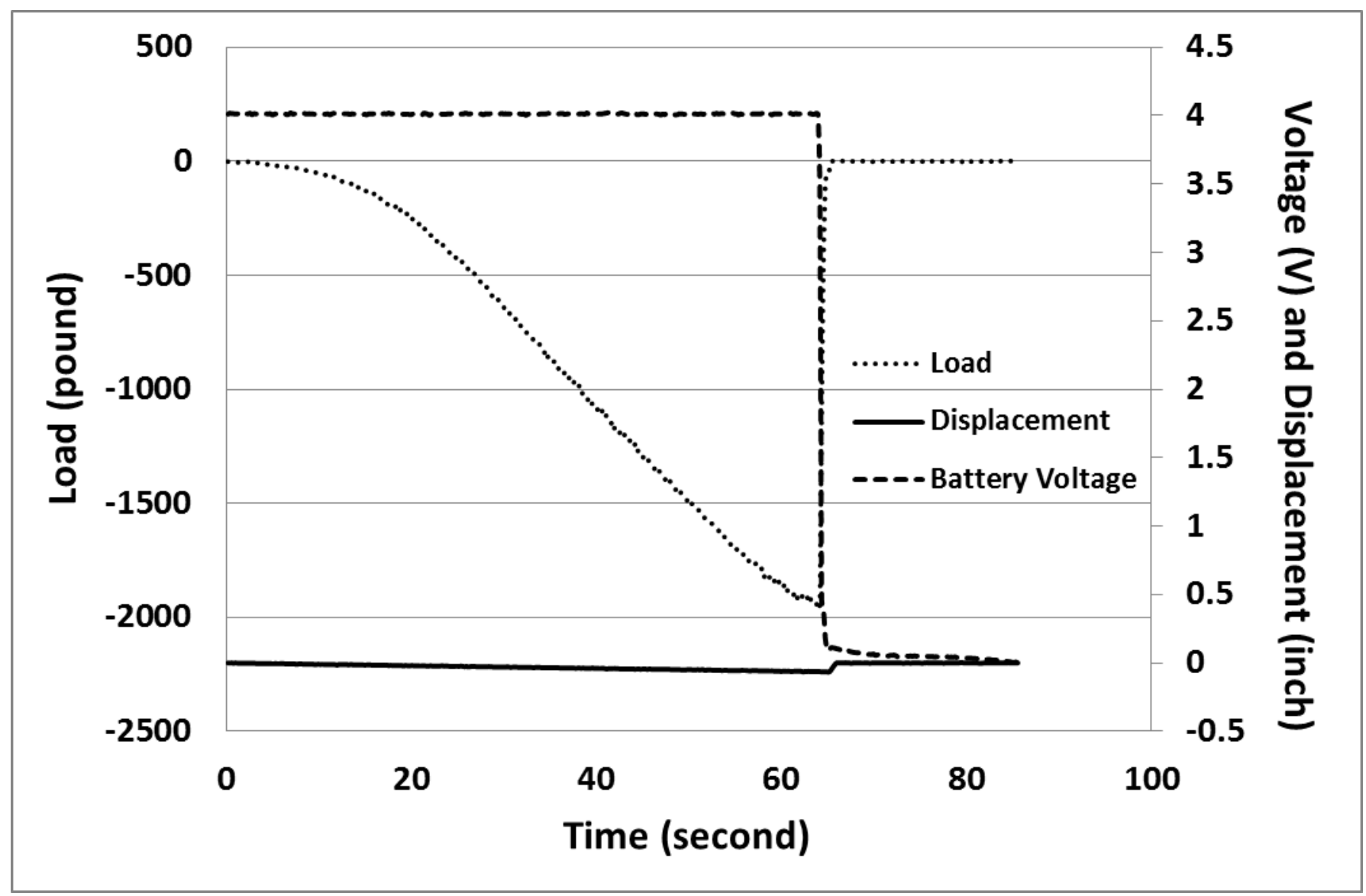

Figure 2. Typical pinch test results using 1-inch diameter steel balls 


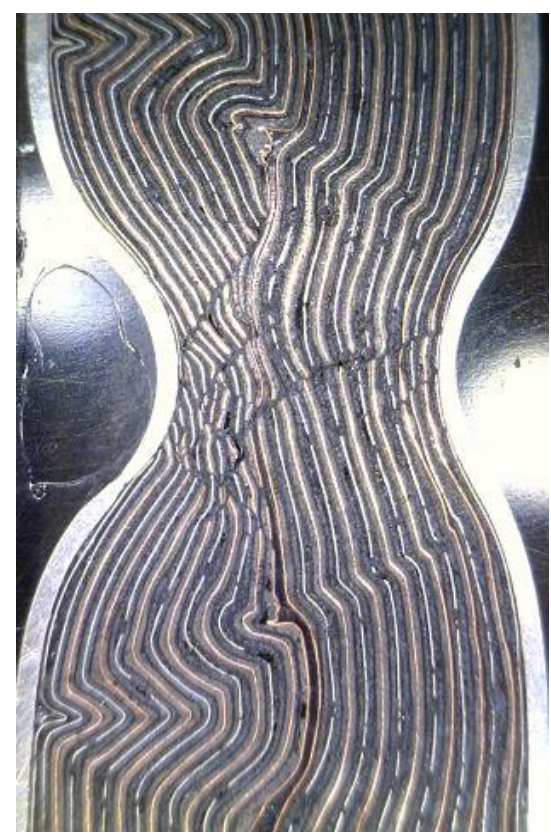

(a)

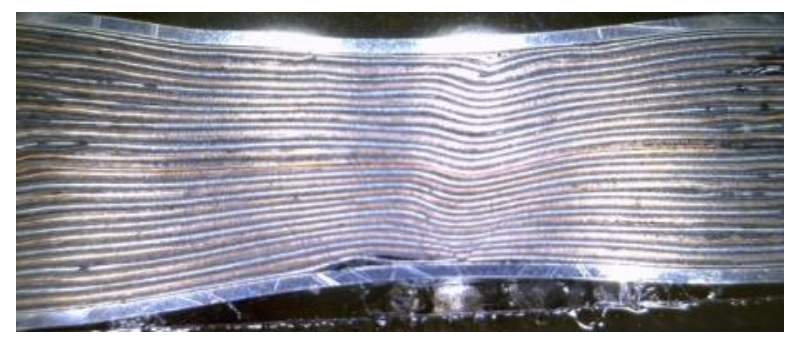

(d)

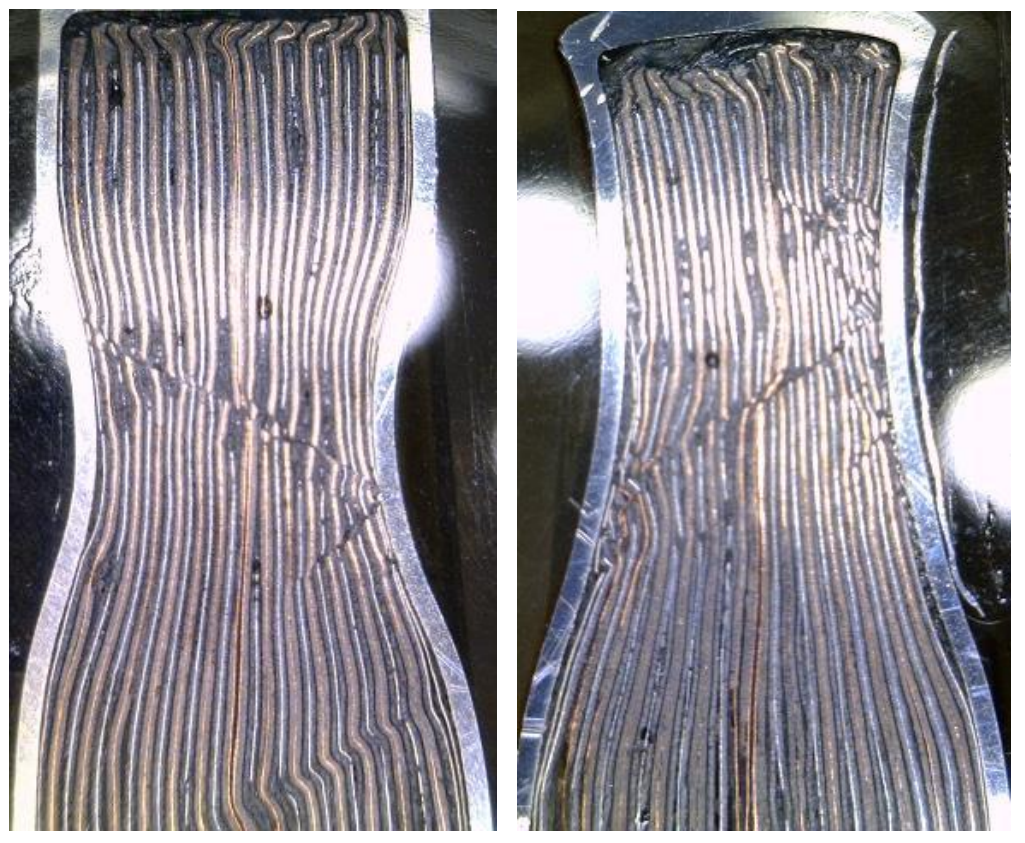

(b)

(c)

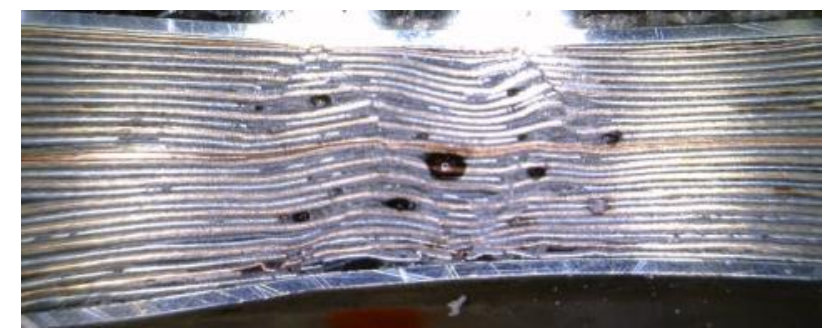

(e)

Figure 3. Internal deformation of Li-ion cells after $0.25 ", 0.5$ ", 1', 2" and 3' diameter pinch tests.

The total cell thickness (including the aluminum can) is $4.3 \mathrm{~mm}$ in all pictures. 

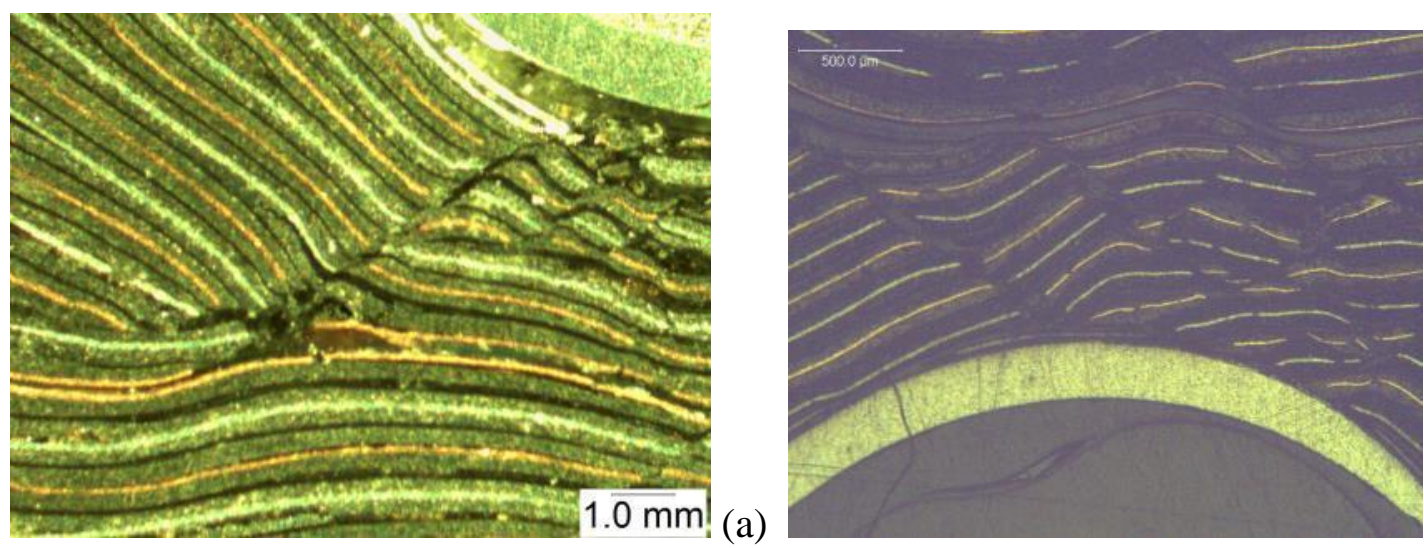

(b)

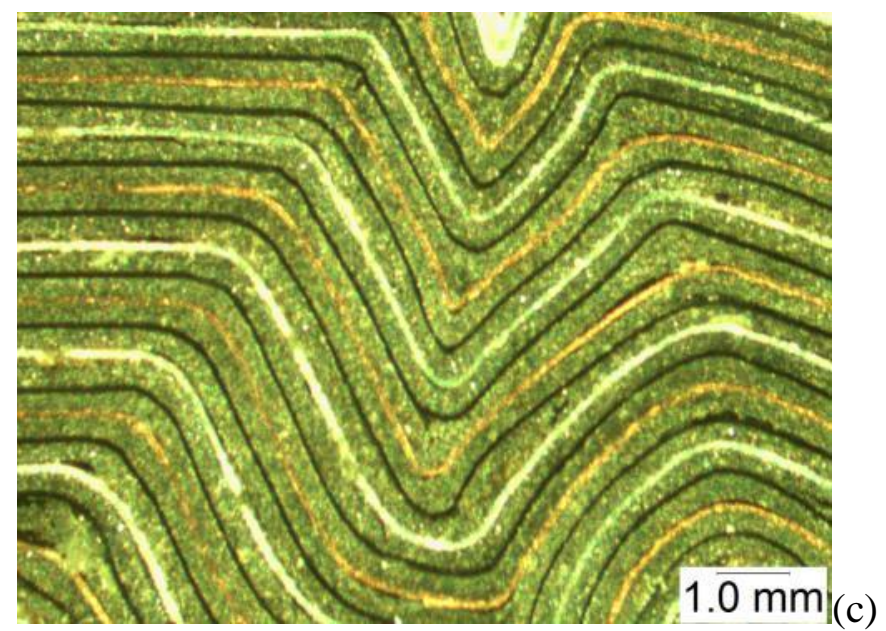

Fig. 4. Three cross section images of Li-ion cell pinched using 0.25 " diameter spheres 


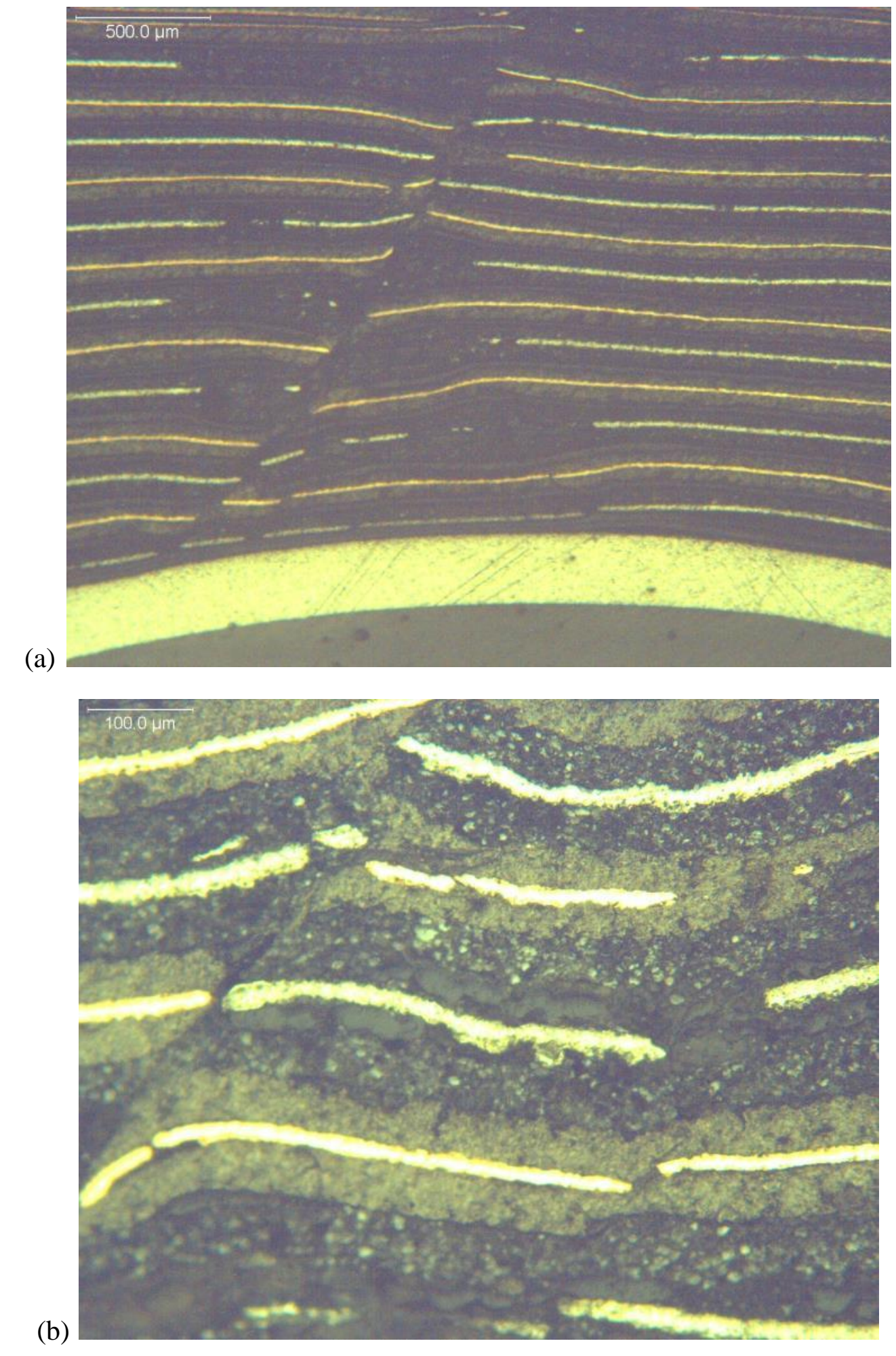

Fig. 5. Two cross section images of Li-ion cell pinched using 0.5 " diameter spheres 

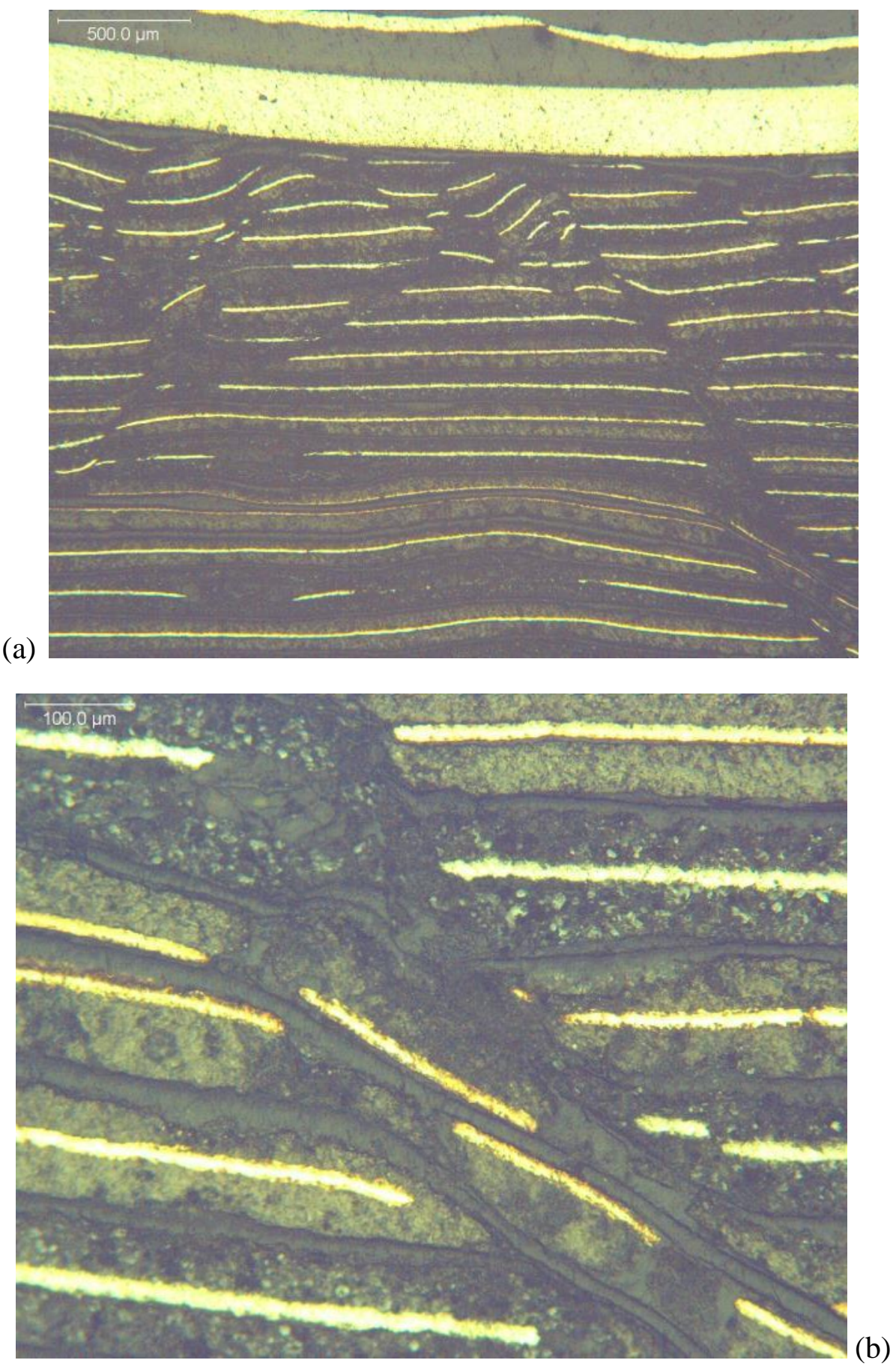

Fig. 6. Two cross section images of Li-ion cell pinched using 1" diameter spheres 

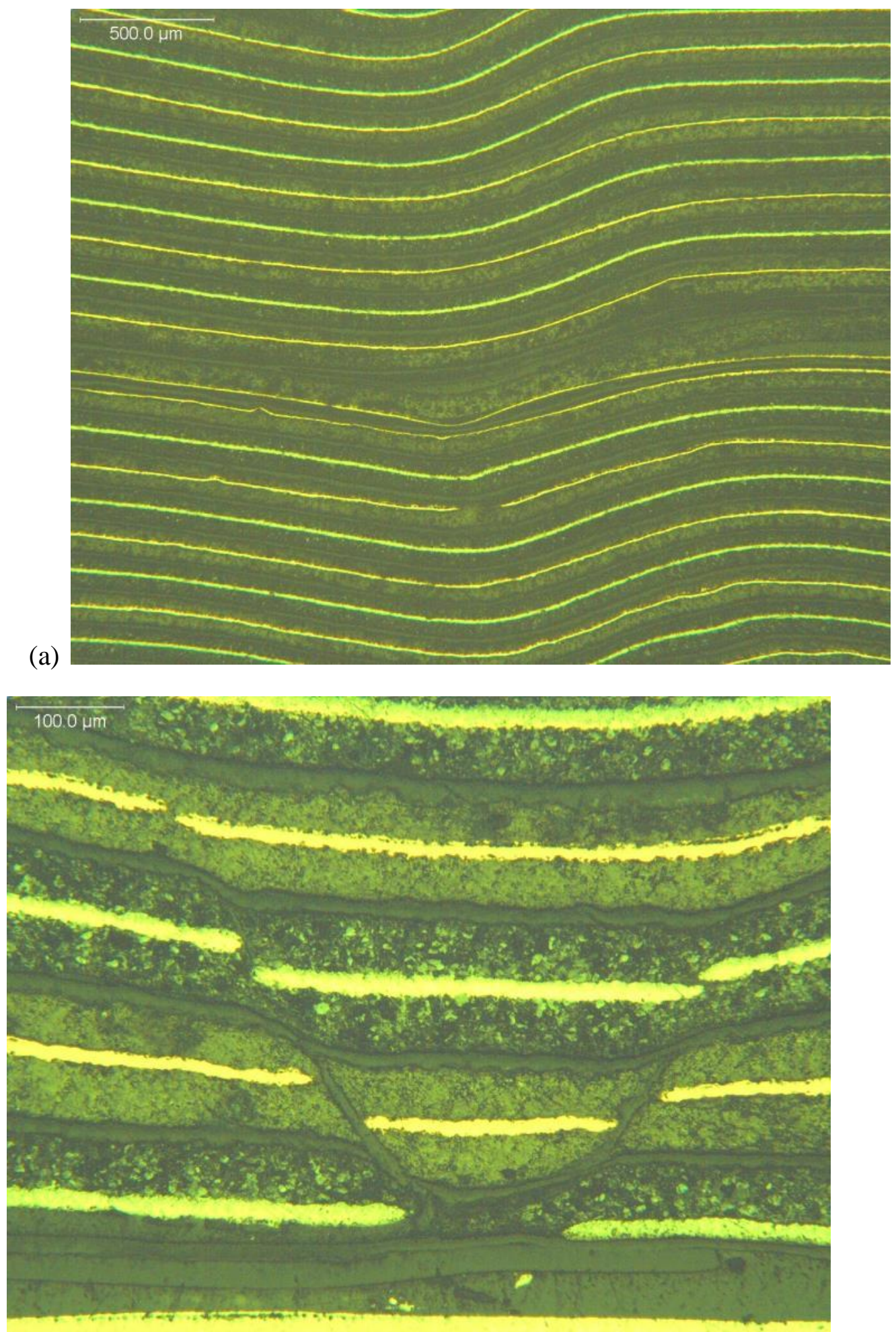

(b)

Fig. 7. Two cross section images of Li-ion cell pinched using 2" diameter spheres 
(a)
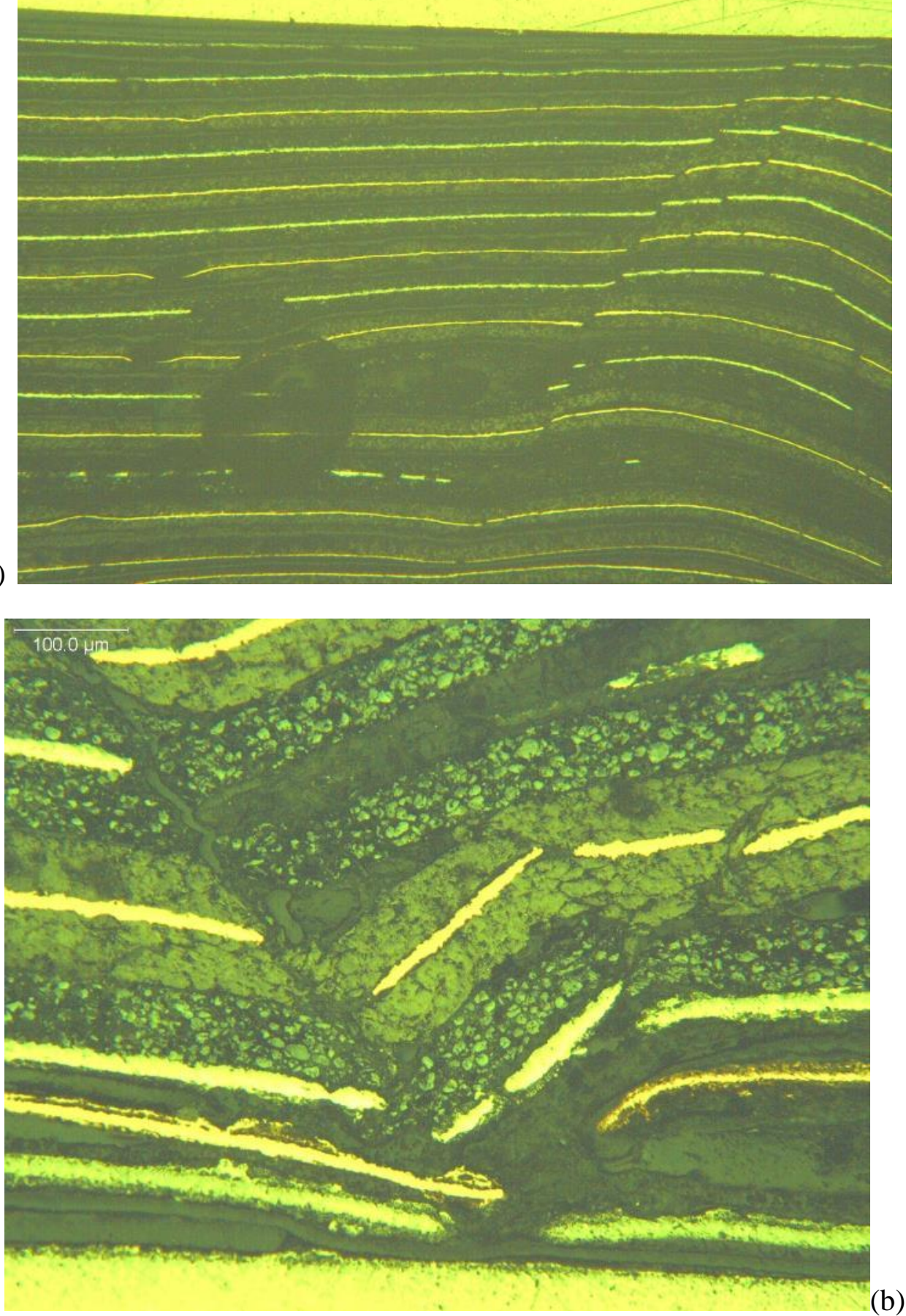

Fig. 8. Two cross section images of Li-ion cell pinched using 3" diameter spheres 